\title{
A Simple Laminar Boundary Layer with Secondary Flow
}

\author{
HENK G. LOOS $\dagger$ \\ California Institute of Technology
}

\section{SUMMARY}

The incompressible laminar boundary layer over a flat plate is studied for the simple case where the stream lines in the free flow have a parabolic shape. An exact solution of the boundarylayer equations is derived. No separation occurs, even when there is a strong adverse pressure gradient along the stream lines, so that in this instance the secondary flow has a favorable influence. Because of the variation of total pressure from one stream line to another in the free stream, the total pressure within the boundary layer at a given point can exceed that of the corresponding free stream.

\section{SYMBoLs}

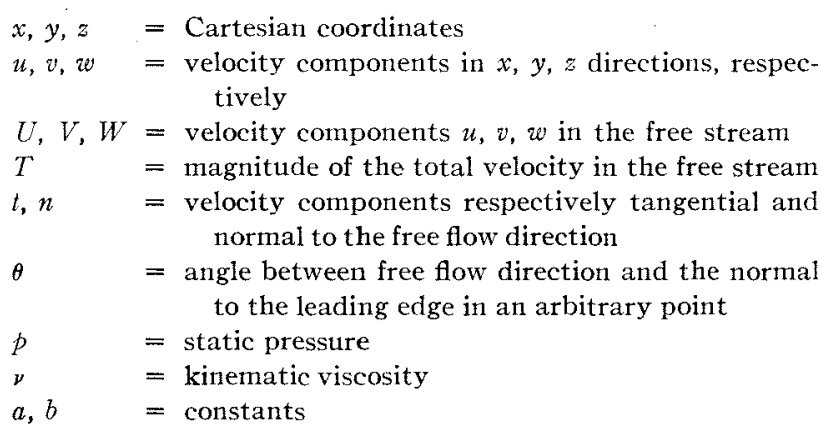

\section{INTRODUCTION}

W HEN AN AIR STREAM FLOWS over the surface of a flat plate, the curvature of the stream lines within the boundary layer may differ from that of the free stream because of a gradient of pressure normal to the direction of free-stream flow. Then, since according to boundary-layer theory the pressure in the bounary layer does not vary with distance from the surface, the boundary-layer flow must, because of its lower velocity, curve more sharply than the free-stream flow in order to balance this pressure gradient. The boundarylayer velocity component normal to the direction of the free stream has become known as the cross flow or the secondary flow. This secondary flow arising from a boundary layer operating in an externally produced pressure gradient was initially discussed by Prand ${ }^{1}$

Received February 22, 1954.

* This work was carried out under the sponsorship of the Office of Scientific Research, Air Research and Development Command and represents a portion of an investigation into secondary flow in axial compressors. The author wishes to express his appreciation to Drs. H. S. Tsien and F. E. Marble for many stimulating discussions.

$\uparrow$ Research Fellow in Jet Propulsion, Daniel and Florence Guggenheim Jet Propulsion Center. and is of considerable importance to the understanding of complex flow phenomena that occur in turbomachinery.

A detailed investigation of the cross flow in the laminar boundary layer on an infinite swept wing was carried out by Sears, ${ }^{2}$ where the pressure gradient in the free stream was normal to the leading edge and, hence, had a component normal to the free-stream flow. Sears calculated the resulting cross flow and showed in particular that the cross flow vanishes for the swept flat plate at zero angle of attack - that is, when the imposed pressure gradient vanished. The cross flow generated by the centrifugal pressure gradient on a propeller or turbomachine blade was investigated in a similar manner by Fogarty, ${ }^{3}$ where the blade was treated as an airfoil of infinite span rotating about a point of its span.

The study of three-dimensional boundary layers has been pursued further by Howarth ${ }^{4}$ and other workers. A problem of particular interest in the study of turbomachines was discussed by Mager and Hansen, ${ }^{5}$ who considered the cross flow generated by a curved free stream, carrying its own pressure gradient, which flows over a semi-infinite flat plate. When the free stream is a potential vortex, a solution was obtained which is valid over distances from the leading edge for which the turning angle of the free stream is small.

Now it seems clear that a considerable portion of the cross flow in boundary layers is directly related to the fact that the velocity in the boundary layer is less than in the free stream. For example, if a thick boundary layer is built up by a straight uniform flow over a plate and then at some point the free stream is turned, the cross flow that takes place has little to do with local viscous stresses but is simply a matter of transporting the vorticity that was generated far upstream. This idea was used by Squire and Winter ${ }^{6}$ to discuss the secondary flow that occurs in airfoil cascades having a thick wall boundary layer and by $\mathrm{Hawthorne}^{7}$ in his treatment of the flow in pipe bends. The process becomes simpler when viscous stresses may be neglected, and it has often been suggested (e.g., Hayes ${ }^{8}$ ) that the general problem of three-dimensional boundary-layer flow may be considered in two parts: an inviscid outer part matched with a viscous sublayer.

The present work, however, is an exact calculation of the laminar boundary layer developed on a flat plate by a flow having stream lines parallel to the plane of the 
plate and of parabolic shape in this plane. The free stream is rotational, having a constant vorticity that is directed normal to the plate. This situation is similar to that which occurs in some problems of fluid motion past turbomachine blades.

\section{Boundary Layer with Parabolic Main Stream}

Let $U, V$, and $W$ denote the components of freestream velocity in the $x, y$, and $z$ directions, respectively, where $x$ is the distance measured along a semiinfinite flat plate in a direction normal to its leading edge, $y$ normal to the plane of the plate, and $z$ normal to these two coordinates as shown in Fig. 1. If the velocity components are chosen to be

$$
\left.\begin{array}{l}
U=\text { constant } \\
V=0 \\
W=a+b x
\end{array}\right\}
$$

with $a$ and $b$ constants, then the stream lines of the main flow are of parabolic shape and the flow is of uniform vorticity, $\Omega=b$, with the vorticity vector in direction of the $y$ axis. The Helmholtz relation for forticity is obviously satisfied.

The boundary-layer equations for an incompressible fluid are, in this case, where the velocity is independent of $z$,

$$
\begin{gathered}
u \frac{u \partial}{\partial x}+v \frac{\partial u}{\partial y}=\nu \frac{\partial^{2} u}{\partial y^{2}} \\
\partial p / \partial y=0 \\
u \frac{\partial w}{\partial x}+v \frac{\partial w}{\partial y}-b U=\nu \frac{\partial^{2} w}{\partial y^{2}} \\
(\partial u / \partial x)+(\partial v / \partial y)=0
\end{gathered}
$$

with the boundary conditions

$$
\begin{gathered}
y=0, \quad\left\{\begin{array}{l}
u=0 \\
v=0 \\
w=0
\end{array}\right. \\
y=\infty, \quad\left\{\begin{array}{l}
u=U \\
w=a+b x
\end{array}\right.
\end{gathered}
$$

Eqs. (2) and (5), together with the boundary conditions for the velocity $u$, are identical with those for the twodimensional laminar boundary layer on a flat plate with zero pressure gradient. Therefore the solution for $u$ and $v$ is the well-known Blasius solution. ${ }^{9}$ Eq. (4) and the boundary conditions for $w$ remain for determination of the velocity component $w$.

Eq. (4) can be reduced to an ordinary differential equation by using $\eta=y \sqrt{U / \nu x}$ as new independent variable. The Blasius solution may be expressed in the form

$$
\begin{aligned}
& u=U F^{\prime}(\eta) \\
& v=\frac{1}{2} \frac{U}{x} \sqrt{\frac{\nu x}{U}} g(\eta)
\end{aligned}
$$

$$
g(\eta)=\int_{0}^{\eta} \eta_{1} F^{\prime \prime}\left(\eta_{1}\right) d \eta_{1}
$$

Therefore if the velocity component parallel to the leading edge is assumed to be of the form

$$
w=a w_{0}(\eta)+b x w_{1}(\eta)
$$

Eq. (4) may be transformed to

$$
\begin{gathered}
(a / x)\left[-(1 / 2)\left(F^{\prime} \eta-g\right) w_{0}^{\prime}-w_{0}^{\prime \prime}\right]+ \\
b\left[-1+F^{\prime} w_{1}-(1 / 2) \times\right. \\
\left.\left(F^{\prime} \eta-g\right) w_{1}^{\prime}-w_{1}^{\prime \prime}\right]=0
\end{gathered}
$$

Furthermore, by partial integration of Eq. (10), the quantity $F^{\prime} \eta-g$ appearing in Eq. (12) may be written

$$
F^{\prime} \eta-g=\int_{0}^{\eta} F^{\prime}\left(\eta_{1}\right) d \eta_{1}
$$

so that Eq. (12) becomes

$$
\begin{aligned}
& (a / x)\left[-(1 / 2) F w_{0}^{\prime}-w_{0}^{\prime \prime}\right]+ \\
& b\left[-1+F^{\prime} w_{1}-(1 / 2) F w_{1}^{\prime}-w_{1}^{\prime \prime}\right]=0
\end{aligned}
$$

Now if the form assumed in Eq. (11) is valid, Eq. (13) must be an identity in $x$ and the quantities $w_{0}$ and $w_{1}$ fulfill the equations

$$
\begin{gathered}
w_{0}^{\prime \prime}+(1 / 2) F w_{0}^{\prime}=0 \\
w_{1}^{\prime \prime}+(1 / 2) F w_{1}^{\prime}-F^{\prime} w_{1}=-1
\end{gathered}
$$

with boundary conditions

$$
\begin{array}{ll}
\eta=0, & \left\{\begin{array}{l}
w_{0}=0 \\
w_{1}=0
\end{array}\right. \\
\eta=\infty, & \left\{\begin{array}{l}
w_{0}=1 \\
w_{1}=1
\end{array}\right.
\end{array}
$$

The Blasius solution for the velocity $u=U F^{\prime}(\eta)$ in the direction of $x$ satisfies the differential equation $F^{\prime \prime} F+$ $2 F^{\prime \prime \prime}=0$, with the boundary conditions $\eta=0, F^{\prime}(0)$; $\eta=\infty, F^{\prime}=1$. It is obvious that the function $w_{0}=$ $F^{\prime}(\eta)$ satisfies Eq. (14) and the boundary conditions [Eqs. (16) and (17)]. The cross-flow component $w_{0}$, which is related with the constant free-flow component $W=a$, has the same profile as the velocity component $u$. This result was first obtained by Sears in reference 2. It means that in the case of a parallel stream approaching a flat plate at an arbitrary angle, the flow direction in the boundary layer is the same as in the free stream. In other words, no cross flow occurs, and, consequently, a cross flow in a boundary layer can only exist if there is a pressure gradient.

\section{Calculation of the Cross Flow}

The Eq. (15) is linear and nonhomogenous; the coefficients $F(\eta)$ and $F^{\prime}(\eta)$ are tabulated functions. ${ }^{9}$ Instead of calculating the solution of Eq. (15) with boundary conditions given by Eqs. (16) and (17), $u$ and $w$ will be related with velocity components $t$ and $n$, which are, respectively, tangential and normal to the 

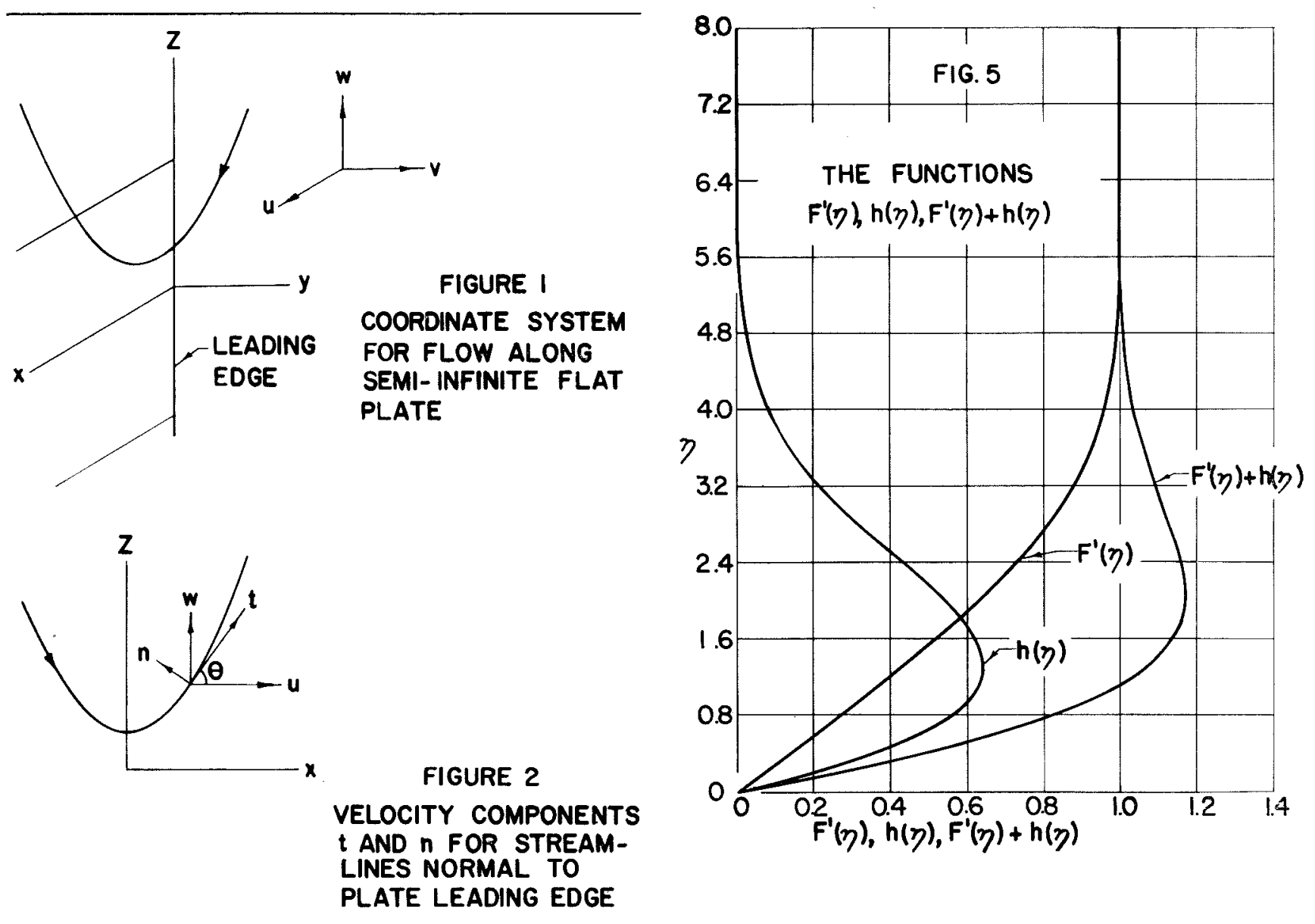

LINES NORMAL TO

PLATE LEADING EDGE

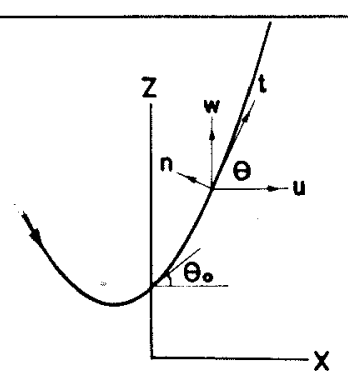

FIGURE 3

VELOCITY COMPONENTS $t$ AND $n$ FOR STREAM-

LINES MEETING LEADING EDGE AT ANGLE $90^{\circ}-\theta$.
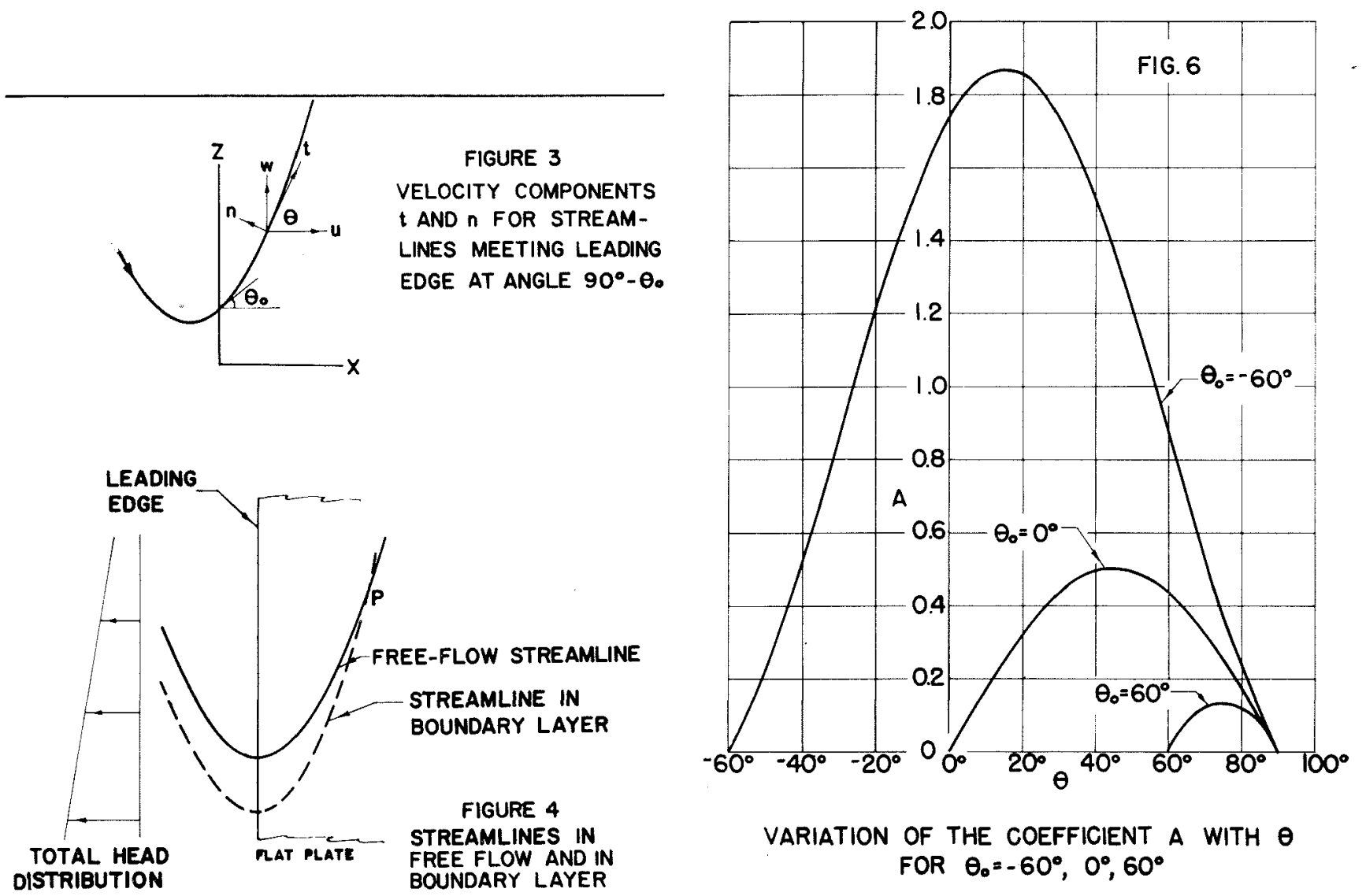

VARIATION OF THE COEFFICIENT A WITH $\theta$ FOR $\theta_{0}=-60^{\circ}, 0^{\circ}, 60^{\circ}$ 
local free-flow direction. In Fig. 2 à free-flow stream line is shown for the case that the coefficient $a$ is zerothat is, so that the stream lines are perpendicular to the leading edge of the plate. If $\vartheta$ is the angle between the free-flow direction and the positive $x$ axis,

$$
\begin{aligned}
& t=u \cos \vartheta+w \sin \vartheta \\
& n=w \cos \vartheta-u \sin \vartheta
\end{aligned}
$$

Now, for $a=0, u=U F^{\prime}(\eta)$ and $w=b x w(\eta)$, and $T=\sqrt{U^{2}+b^{2} x^{2}}$ is the local free-stream velocity. Then Eqs. (18) and (19) may be written as

$$
\begin{gathered}
\frac{t}{T}=\frac{u^{2} F^{\prime}+b^{2} x^{2} w_{1}}{u^{2}+b^{2} x^{2}}=\frac{F^{\prime}+(b x / u)^{2} w_{1}}{1+(b x / U)^{2}} \\
\frac{n}{T}=\frac{b x U w_{1}-b x U F^{\prime}}{U^{2}+b^{2} x^{2}}=\frac{b x}{U} \frac{w_{1}-F^{\prime}}{1+(b x / U)^{2}}
\end{gathered}
$$

Now it is convenient to define

$$
h(\eta)=w_{1}(\eta)-F^{\prime}(\eta)
$$

Then a differential equation for $h(\eta)$ can be derived in the following way: The function $w_{1}(\eta)$ is a solution of $w_{1}^{\prime \prime}+(1 / 2) F w_{1}^{\prime}-F^{\prime} w_{1}=-1$, and $F(\eta)$ satisfies the equation $F^{\prime \prime \prime}+(1 / 2) F F^{\prime \prime}=0$. Subtraction of these differential equations gives

$$
\begin{aligned}
\left(w_{1}-F^{\prime}\right)^{\prime \prime}+F\left(w_{1}-F^{\prime}\right)^{\prime}-F^{\prime}\left(w_{1}-F^{\prime}\right)= & \\
-1 & +\left(F^{\prime}\right)^{2}
\end{aligned}
$$

and taking account of the definition of $h(\eta)$,

$$
h^{\prime \prime}(\eta)+(1 / 2) F h^{\prime}-F^{\prime} h=-1+\left(F^{\prime}\right)^{2}
$$

The function $h(\eta)$ is equal to $-G^{\prime}(\eta)$ which has been tabulated by Mager and Hansen. ${ }^{5}$ The normal or cross-flow component [Eq. (21)], may now be written as

$$
\frac{n}{T}=\frac{b x}{U} \frac{h(\eta)}{1+(b x / U)^{2}}
$$

For small values of $x,(b x / U)^{2} \ll 1$, and the normal flow component is the same as that found by Mager and Hansen ${ }^{5}$ for small turning angles. This result is obvious, because, for a flow with $a=0$, the circular stream line coincides in first order with a parabolic stream line for points close to the leading edge.

From Eq. (24) it follows that for large turning angles in "parabolic flow" the distribution of the relative normal flow component $n / T$ remains the same and the magnitude varies as $(b x / U) /\left[1+(b x / U)^{2}\right]$. It is possible to express $n / T$ in terms of the turning angle $\vartheta$ of the free flow. For $a=0, b x / U=\tan \vartheta$ and

$$
(b x / U) /\left[1+(b x / U)^{2}\right]=(1 / 2) \sin 2 \vartheta
$$

and therefore

$$
n / T=(1 / 2) h(\eta) \sin 2 \vartheta
$$

Further, Eq. (20) can be written as

$$
t / T=F^{\prime}(\eta)+h(\eta) \sin ^{2} \vartheta
$$

In the general case where $a \neq 0, w=a w_{0}(\eta)+$ $b w_{1}(\eta)$, the normal velocity component can be expressed as

$$
n=\left[a F^{\prime}+b x\left(F^{\prime}+h\right)\right] \cos \vartheta-U F^{\prime} \sin \vartheta
$$

Now, $\tan \vartheta=(a+b x) / U$, so that

$$
n=b x h(\eta) \cos \vartheta
$$

Further, the quantity $b x$ may be written $b x=U(\tan$ $\vartheta-\tan \vartheta_{0}$ ), where $\vartheta_{0}$ is the angle between the free-flow direction and the $x$ axis at the leading edge (see Fig. 3). Eq. (27) becomes

$$
\begin{gathered}
n / T=A\left(\vartheta, \vartheta_{0}\right) h(\eta) \\
A\left(\vartheta, \vartheta_{0}\right)=\cos ^{2} \vartheta\left(\tan \vartheta-\tan \vartheta_{0}\right)
\end{gathered}
$$

In the same way an expression for $t / T$ may be derived in terms of $F^{\prime}(\eta), h(\eta), \vartheta$, and $\vartheta_{0}$,

$$
\begin{gathered}
t / T=F^{\prime}(\eta)+B\left(\vartheta, \vartheta_{0}\right) h(\eta) \\
B\left(\vartheta, \vartheta_{0}\right)=(1 / 2) \sin 2 \vartheta\left(\tan \vartheta-\tan \vartheta_{0}\right)
\end{gathered}
$$

\section{RESULTS}

The functions $A\left(\vartheta, \vartheta_{0}\right)$ and $B\left(\vartheta, \vartheta_{0}\right)$ are plotted in Figs. 6 and 7 for $\vartheta_{0}=-60^{\circ}, 0^{\circ}$, and $60^{\circ}$. For the same values of $\vartheta_{0}$, the tangential flow $t / T$ is plotted versus $\eta$ for some values of $\vartheta_{0}$

In Fig. 8, $\vartheta_{0}=-60^{\circ}$ and $\vartheta=-60^{\circ},-40^{\circ} \ldots 60^{\circ}$. For $\vartheta=-40^{\circ}$ and $\vartheta=-20^{\circ}$, a "separation" type profile is found, caused by the adverse pressure gradient along the direction of the free-stream flow. As will be shown later, the cross flow prevents any actual separation.

After passing the point $\vartheta=0$, the velocity profile becomes fuller and at the point $\vartheta=20^{\circ}$ the velocity in the upper part of the boundary layer exceeds the free-stream velocity by a small amount. For greater values of $\vartheta$, the maximum velocity in the boundary layer increases more and more, until at $\vartheta=60^{\circ}$ the velocity distribution with the highest velocity peak is reached. For $\vartheta>60^{\circ}$, the maximum velocity in the boundary layer drops again, and in the limit $\vartheta=90^{\circ}$ the velocity distribution is just $F^{\prime}(\eta)+h(\eta)$, still having an increased velocity in the upper part of the boundary layer. For $\vartheta_{0}=0^{\circ}$ and $\vartheta_{0}=60^{\circ}$ (Figs. 9 and 10) the velocity profile becomes fuller with increasing $\vartheta$, and the highest velocity peak in the boundary layer is reached at $\vartheta=90^{\circ}$. Of course, for this value of $\vartheta_{0}$, no "separation" type profile occurs because there is no adverse pressure gradient for the tangential flow. The normal or cross flow $n / T$ is distributed over $\eta$ as $A(\vartheta$, $\left.\vartheta_{0}\right) h(\eta)$, and so the velocity profile will be always of the shape $h(\eta)$ (see Fig. 5) while the amplitude changes as $A\left(\vartheta, \vartheta_{0}\right)$ (see Fig. 6).

It is of interest to investigate the total head distribution in the boundary layer. Because, in the boundarylayer approximation, the pressure is constant through the boundary layer, the total head loss in the boundary 
A SIMPLE LA M I N R BOUNDAR Y LAYER WITH SECONDAR F F OW 39
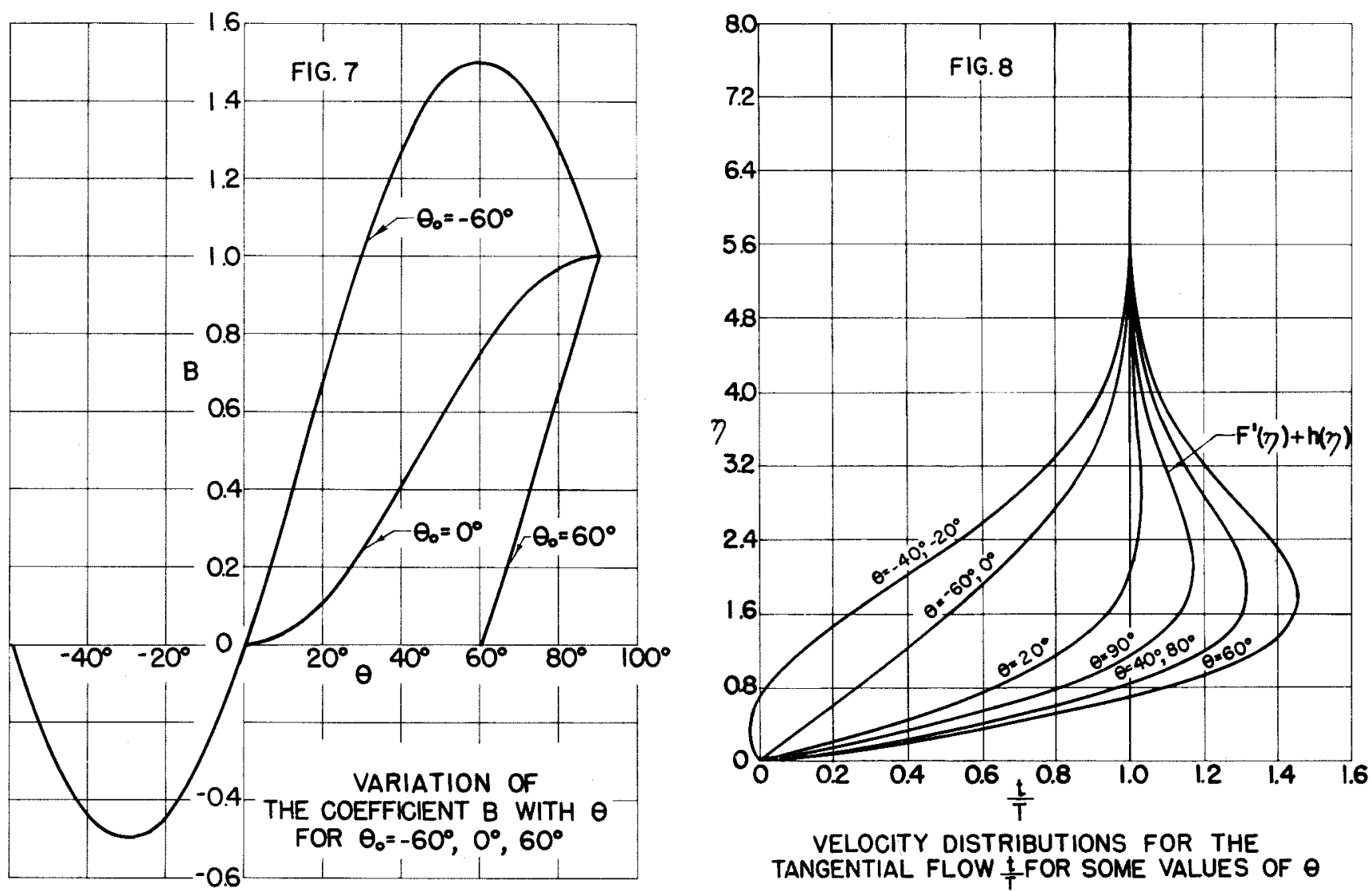

VELOCITY DISTRIBUTIONS FOR THE TANGENTIAL FLOW $\frac{t}{T} F O R$ SOME VALUES OF $\theta$ AND FOR $\theta_{0}=-60^{\circ}$

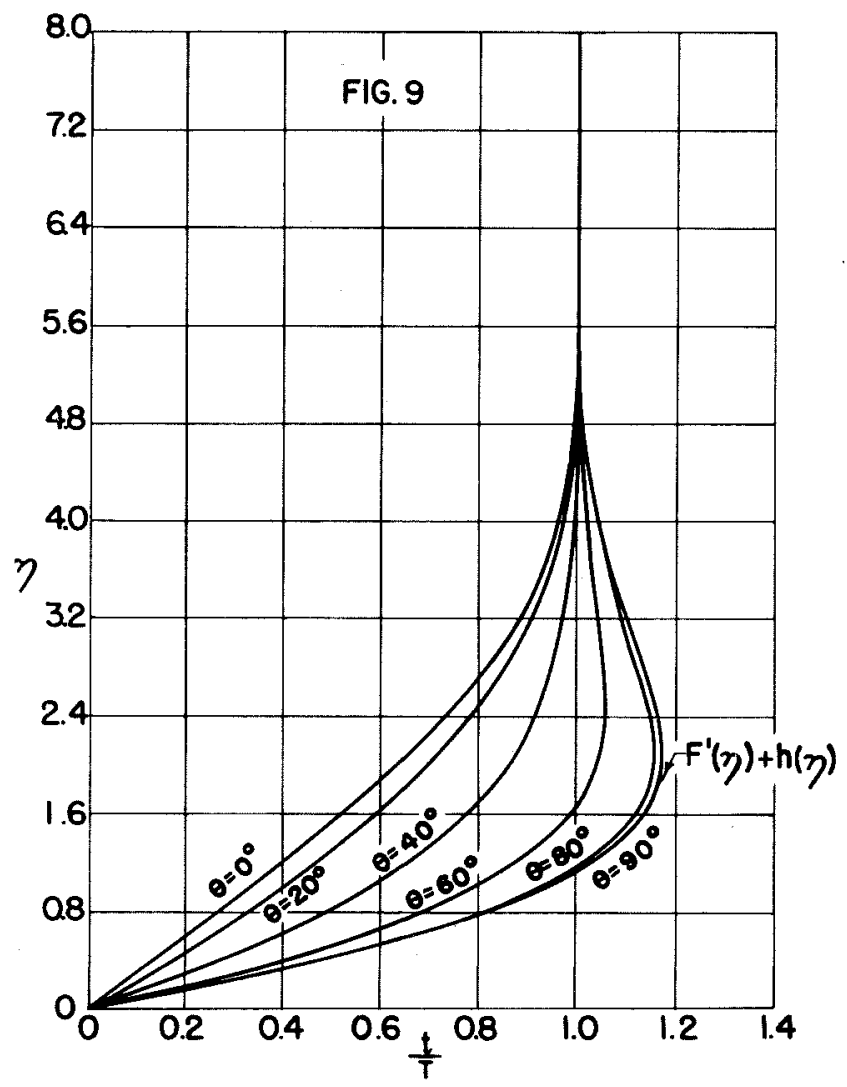

VELOCITY DISTRIBUTIONS FOR THE

TANGENTIAL FLOW $\frac{t}{T}$ FOR SOME VALUES OF $\theta$ AND FOR $\theta_{0}=0^{\circ}$

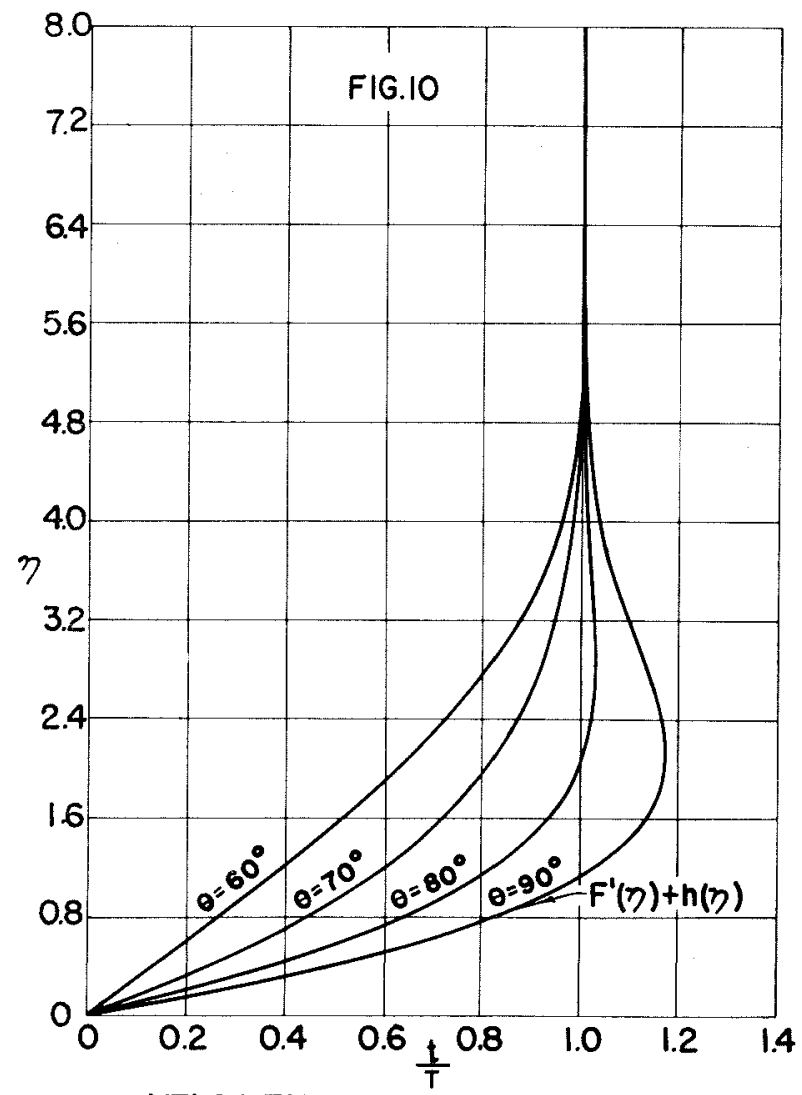

VELOCITY DISTRIBUTIONS FOR THE TANGENTIAL FLOW $\frac{t}{T}$ FOR SOME VALUES OF $\theta$ AND FOR $\theta_{0}=60^{\circ}$ 
layer can be determined by calculating the kinetic energy. Neglecting the velocity component $v$, the ratio of the kinetic energy in the boundary layer and in the free flow is, for $\vartheta_{0}=0$,

and with

$$
\frac{E(\eta)}{E(\infty)}=\frac{\left(U F^{\prime}\right)^{2}+\left(F^{\prime}+h\right)^{2} b^{2} x^{2}}{U^{2}+b^{2} x^{2}}
$$

$$
\begin{gathered}
U / \sqrt{U^{2}+b^{2} x^{2}}=\cos \vartheta, b x / \sqrt{U^{2}+b^{2} x^{2}}=\sin \vartheta \\
E(\eta) / E(\infty)=\left(F^{\prime}\right)^{2} \cos ^{2} \vartheta+\left(F^{\prime}+h\right)^{2} \sin ^{2} \vartheta
\end{gathered}
$$

If the ratio $E(\eta) / E(\infty)$ exceeds unity, the total head at the point of the boundary layer exceeds the freestream value. If $\eta=2.0$ is chosen, according to Fig. $5, F^{\prime}(2.0)=0.63$ and $F^{\prime}(2.0)+h(2.0)=1.17$. Thus if

$$
E(2.0) / E(\infty)=0.63^{2} \cos ^{2} \vartheta+1.17^{2} \sin ^{2} \vartheta>1
$$

that is, if $\vartheta>52.1^{\circ}$, the energy in the boundary layer at $\eta=2.0$ exceeds the free-stream energy.

This result can be understood by comparing the stream lines of the free stream and of the boundary layer (Fig. 4). Since the stream lines in the boundary layer curve more sharply than those in the free stream, the stream line passing the observation point $P$ at $\eta=$ 2.0 (dashed line in Fig. 4) comes from another upstream location than the free-flow stream line passing through the same point $P$. There is a total head gradient in the $z$ direction upstream of the leading edge because of the vorticity in the parabolic flow, so that upstream of the leading edge the dashed stream line through $P$ will have a larger total head. Along the dashed stream line, energy will be dissipated, but it is possible that the energy loss is less than the difference in total head upstream of the leading edge. In this case, the total head in some point of $P$ will exceed the free-stream value.

Another interesting question is whether there will be a separation of the boundary layer. Of course, separation cannot be expected for $\vartheta_{0}>0$ because the pressure gradient in this case is favorable. But if $-\vartheta_{0}$ is sufficiently large, an adverse pressure gradient occurs in the region $\vartheta_{0}<\vartheta<0$, and if there were no cross flow, the boundary layer would separate. Actually, a cross flow occurs, and the separation may be studied by employing the solution for the boundary-layer flow which has been obtained.

If three-dimensional separation occurs, then, according to Hayes, ${ }^{8}$ there is a separation line on the surface which is tangent to all "stream lines" at the surface. In the present example, the velocity field is constant in the $z$ direction, so that any separation line must be parallel with the $z$ axis. The flow direction $\vartheta^{\prime}$ on the surface is given by

$$
\begin{aligned}
\tan \vartheta^{\prime}=\lim _{y \rightarrow 0} \frac{w}{u} & =\lim _{y \rightarrow 0} \frac{a F^{\prime}+b x\left(F^{\prime}+h\right)}{U F^{\prime}} \\
& =\frac{a+b x\left[1+\lim _{\eta \rightarrow 0} h(\eta) / F^{\prime}(\eta)\right]}{U}
\end{aligned}
$$

Now

$$
\lim _{\eta \rightarrow 0} h(\eta) / F^{\prime}(\eta)
$$

is finite so that, for finite values of $x, \tan \vartheta^{\prime}$ will be finite. Therefore no separation occurs for finite values of $x$. From this result it follows that the cross flow avoids separation in the present example by transporting fluid with high energy to the "critical" regions.

\section{CONCLUSIONS}

The boundary-layer equations for the "parabolic" flow along a flat plate can be solved exactly, giving information about the secondary flow (cross flow). The solution for $\vartheta_{0}=0$ coincides with that of Mager and Hansen $^{5}$ for small turning angles but also remains valid for large angles. There, the formulas of Mager and Hansen can be used, replacing the turning angle $\vartheta$ by $(1 / 2) \sin 2 \vartheta$.

If $-\vartheta_{0}$ is large enough, a "separation" type profile for the tangential flow will be found, but there will be no separation because the secondary flow transports fluid with high energy to the "critical" regions. According to the total head gradient upstream of the leading edge (rotation), the total head in the boundary layer can exceed the free-stream value.

\section{REFERENCES}

' Prandt1, L., On Boundary Layers in Three Dimensional Flow. M.A.P. Volkenrode VG 89.

${ }^{2}$ Sears, W. R., The Boundary Layer of Yawed Cylinders, Journal of the Aeronautical Sciences, Vol. 15, No. 1, p. 49, January, 1948.

${ }^{3}$ Fogarty, L. E., The Laminar Boundary Layer on a Rotating Blade, Journal of the Aeronautical Sciences, Vol. 18, No. 4, pp. 247-252, April, 1951.

"Howarth, L., The Boundary Layer in Three Dimensional Flow, Part I, Phil. Mag., Ser. 7, Vol. xlii, May, 1951.

${ }^{5}$ Mager, A., and Hansen, A. G., Laminar Boundary Layer over' Flat Plate in a Flow Having Circular Streamlines, NACA TN. No. 2658, March, 1952.

'Squire, H. B., and Winter, K. G., The Secondary Flow in Cas. cade of Airfoils in a Nonuniform Stream, Journal of the Aeronautical Sciences, Vol. 18, No. 4, pp. 271-277, April, 1951.

' Hawthorne, W. R., Secondary Circulation in Fluid Flow, Proc. of The Royal Society of London, Series A, Vol. 206, No. A1086, pp. 374-387, May, 1951.

${ }^{8}$ Hayes, W. D., The Three Dimensional Boundary Layer. NAVORD Report 1313, NOTS 384, May 9, 1951.

${ }^{9}$ Blasius, H., Grenzschichten in Flilssigkeiten mit kleiner Reibung, Zeitschrift fur Mathematik und Physik, Band 56, p. 1. 1908. 